ISSN 0103-5150

Fisioter. Mov., Curitiba, v. 30, n. 3, p. 607-623, Jul./Sep. 2017

Licenciado sob uma Licença Creative Commons

DOI: http://dx.doi.org/10.1590/1980-5918.030.003.AR02

(c)

\title{
Phisical modalities on the functional performance in knee osteoarthritis: a sytematic review
}

\author{
Modalidades físicas no desempenho funcional na \\ osteoartrite de joelho: uma revisão sistemática
}

\author{
Letícia Ferronato, Hemily Marega Cunha, Pâmela Maiara Machado, Gabriela dos Santos de Souza, \\ Mirieli Denardi Limana, Núbia Carelli Pereira de Avelar*
}

Universidade Federal de Santa Catarina (UFSC), Araranguá, SC, Brazil

\begin{abstract}
Introduction: Despite recent advances in the treatment of osteoarthritis (OA), few studies have evaluated the longitudinal effect of physical modalities in functional capacity in patients with knee OA. Thereby, since the physical components and pain can affect the functional performance of daily activities, the effect of these treatment's form is still to be established. Objective: Evaluate the effectiveness of therapeutic ultrasound, electrical stimulation and phototherapy in the functional performance, in patients with knee osteoarthritis. Methods: Articles present in the PubMed, Lilacs, SciELO and PEDro's databases were evaluated. The used keywords were "pulsed ultrasound therapy", "ultrasound therapy", "electric stimulation" and "low level laser therapy" in combination with "knee osteoarthritis". Were included in this presented review, randomized clinical studies using ultrasound, electrical and laser stimulation in subjects with knee osteoarthritis. To evaluate the methodological quality of the selected studies, was used the PEDro's scale. The dependent variables of the study were: pain, physical function, joint stiffness, life quality and functional performance.
\end{abstract}

\footnotetext{
* LF: BS, e-mail: lety_ferronato@hotmail.com HMC: undergrad, e-mail: hemily_cunha1@hotmail.com PMM: undergrad, e-mail: pamelaamaiara@gmail.com GSS: undergrad, e-mail: ggabrielassouza@gmail.com MDL: MS, e-mail: mirieli.limana@ufsc.br NCPA: PhD, e-mail: nubia.carelli@ufsc.br
} 
Results: 268 studies were found, of these, 41 studies met eligibility criteria and were classified for analysis in full. The used methodology in the studies varied widely, however, in most cases there was improvement in functional performance of individuals with knee $\mathrm{OA}$, with the use of physical modalities, for the pulsed ultrasound, continuous ultrasound, electrical stimulation and laser resourses. Conclusion: The physical modalities used in the studies demonstrated improvement in functional performance of individuals with knee OA.

Keywords: Osteoarthritis. Treatment. Physical Therapy.

\title{
Resumo
}

\begin{abstract}
Introdução: Apesar dos avanços recentes no tratamento da Osteoartrite (OA), poucos estudos avaliaram o efeito longitudinal de modalidades físicas na capacidade funcional em pacientes com OA do joelho. Assim, uma vez que os componentes físicos e dor podem afetar o desempenho funcional de atividades diárias, o efeito dessas formas de tratamento ainda está para ser estabelecido. Objetivo: Avaliar a efetividade do uso do ultrassom terapêutico, estimulação elétrica e fototerapia no desempenho funcional em indivíduos com osteoartrite de joelhos. Métodos: Artigos presentes nas bases de dados PubMed, Lilacs, SciELO e PEDro foram avaliados. As palavras-chave utilizadas foram: "pulsed ultrasound therapeutic", "ultrasound therapeutic", "electric stimulation" e "low level laser therapy" em combinação com "knee osteoarthritis". Foram incluídos na presente revisão, estudos clínicos randomizados que utilizaram ultrassom, estimulação elétrica e laser em indivíduos com OA de joelhos. Para avaliar a qualidade metodológica dos estudos selecionados, utilizou-se a escala PEDro. As variáveis dependentes do estudo foram: dor, função física, rigidez articular, qualidade de vida e desempenho funcional. Resultados: Foram encontrados 268 estudos, desses, 41 estudos atenderam aos critérios de elegibilidade e foram classificados para análise na íntegra. A metodologia utilizada pelos estudos variou amplamente, no entanto, em sua maioria houve melhora no desempenho funcional de indivíduos com OA de joelho com a utilização de modalidades físicas, para os recursos Ultrassom pulsado, ultrassom contínuo, estimulação elétrica e laser. Conclusão: $O$ uso das modalidades físicas utilizadas nos estudos demonstraram melhora no desempenho funcional de indivíduos com OA de joelhos.
\end{abstract}

Palavras-chave: Osteoartrite. Tratamento. Fisioterapia.

\section{Introduction}

Osteoarthritis $(\mathrm{OA})$ is a chronic, progressive and degenerative osteo-articular disease characterized by arthralgia, stiffness and joint function limitation. The OA's etiology involves biomechanical, biochemical and genetic's factors that contribute to the instability between articular cartilage's synthesis and destruction (1) may affect muscle performance (2). The OA mainly affects the joints that support weight discharge and, among them, the knee joint is the most affected $(1,3)$.

The knee OA may affect the activities of daily life, reducing the strength, power and muscular endurance, providing decrease in proprioceptive acuity and body balance. These changes may affect the subjective perception of pain, stiffness and physical function and hinder the performance of functional activities, such as, walking, lifting and sit in a chair and up and down stairs (2).

Despite the availability of treatments provided for patients with knee osteoarthritis, the option is inicially for non pharmacological interventions due to no deleterious side effects and are less aggressive for the patient compared to pharmacological and surgical treatments (4). The objective of non pharmacological treatments, including physical therapy, is the relief of the signs and symptoms of the disease and, if possible, the delay in progression, being that various treatments have been used for this purpose, including therapeutic ultrasound (5), electrical stimulation (5, 6) and the low power laser (7).

Despite recent advances in OA's treatment, few studies have evaluated the longitudinal effect of 
physical modalities in functional capacity in patients with knee $\mathrm{OA}$, especially functional capacity during daily life activities and instrumental activities of daily living (5). Thus, since the physical components and pain can affect the functional performance of daily activities, the effect of these forms of treatment on the functional performance and self perception of the disease status in subjects with knee OA is yet to be established. Therefore, this review aims to evaluate the effectiveness of therapeutic ultrasound, electrical stimulation and phototherapy in the functional performance in patients with knee osteoarthritis.

\section{Methods}

\section{Search strategies}

The following electronic databases were searched from January to March 2014: PubMed, Lilacs, SciELO and PEDro. The keywords used were: "pulsed ultrasound therapy", "ultrasound therapy", "electric stimulation" and "low level laser therapy" in combination with "knee osteoarthritis".

Two evaluators ( $\mathrm{LF}$ and $\mathrm{HC}$ ) independently selected the studies based on titles, excluding those which were not related to this review's subject. After the selection, the evaluators reviewed the summaries of selected articles to identify those which met the inclusion and exclusion criteria of the study to then be analyzed in detail.

Eligibility criteria

The following inclusion criteria were used in this study:

1) Types of studies: only randomized controlled experiments involving application of physical modalities (ultrasound, electrical stimulation and laser) in patients with knee OA were selected.

2) Types of participants: studies involving patients diagnosed with knee OA were selected. The diagnosis was established based on valid instruments, such as the classification criteria of the American College of Rheumatology (8), radiographic or laboratory evidence or medical records. No other restriction on the duration of the disease and intensity were applied.
3) Types of interventions: studies comparing physical modalities (therapeutic ultrasound, electrical stimulation and low-level laser therapy), with groups without treatment (control) or placebo groups.

4) Types of result's measures: the dependent variables of the study were: 1) pain, 2) physical function, or 3) joint stiffness. If available, data on life quality and functional performance, served as secondary outcome measures.

5) Score greater than or equal to 5 on the PEDro scale (9).

We selected randomized controlled experiments published in English, Portuguese or Spanish over the last 10 years.

\section{Selection of studies}

After removal of duplicate titles, summaries of all identified articles were analized by two reviewers. Full articles were then read in detail and the eligible ones were included in this systematic review.

\section{Data collection}

Two reviewers independently extracted data on study characteristics, such as participants, interventions, control conditions, co-interventions, outcome measures and results. Disagreements were analyzed by a third reviewer and resolved by discussion.

\section{Endpoints}

The study endpoints were defined with the use of tests to evaluate the physical and functional performance. The Western Ontario and McMaster Universities Osteoarthritis Index (WOMAC) (10) was used for the evaluation of the self-reported state of osteoarthritis and the Visual Analogue Scale for pain evaluation.

\section{Methodological quality rating}

The PEDro scale (9), which is based on the Delphi list (11), Portuguese translated in 2009, was used for methodological quality evaluation of the studies. 
Were included in the study, articles higher than or equal to 5 , since studies scored equal to or exceeding 5 (50\%) are considered high quality, according to Moseley et al. (12).

\section{Results}

In the bibliographic research they were initially found 268 studies, of these, 148 were excluded because they are not in accordance with the proposed goal from the title or being duplicates, leaving 120 articles for the summary reading. 69 articles were excluded because they did not meet any of the study eligibility criteria, leaving 51 articles for full and detailed reading. Finally, 10 studies were excluded due to lower score than 5 on the PEDro scale. Were included 41 trials, reaching 2442 patients total (Figure 1). The sample size of the 41 studies ranged from 3 to 175 individuals with $\mathrm{OA}$. To facilitate the visualization of the articles included in this review, more detailed results are described in Table 1.

The studies included in this review were divided to discuss the results according to each physical mode. Therefore, of these studies 41, 4 refered to the pulsed therapeutic ultrasound, 7 to the continuous therapeutic ultrasound, 8 neuromuscular electrical stimulation, 12 to transcutaneous electrical nerve stimulation, 3 pulsed electrical stimulation and 7 to the low level laser therapy.

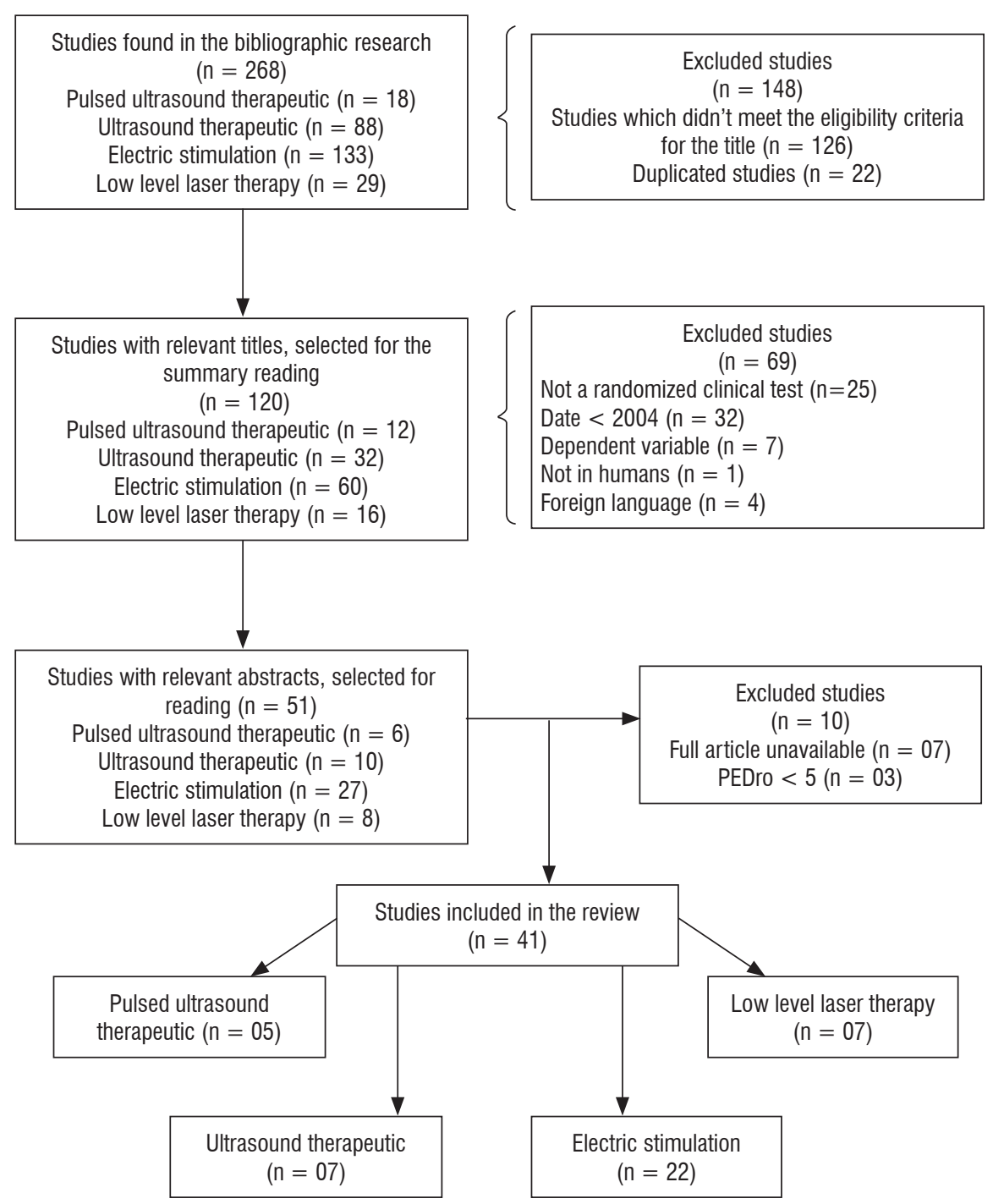

Figure $\mathbf{1}$ - Study selection results for keywords. 
Table 1 - Studies general data

\begin{tabular}{|c|c|c|c|c|c|c|c|}
\hline Study & Sample & Groups & Program & Instruments & $\begin{array}{l}\text { Endpoints } \\
\text { Evaluated }\end{array}$ & Results & PEDro \\
\hline \multicolumn{8}{|c|}{ Therapeutic pulsed ultrasound: } \\
\hline $\begin{array}{l}\text { Tascioglu et } \\
\text { al. (2010) } \\
(13)\end{array}$ & 82 & $\begin{array}{l}\text { CU x PU x } \\
\text { placebo }\end{array}$ & $\begin{array}{l}\mathrm{CU}: 1 \mathrm{MHz}, 2 \mathrm{~W} / \mathrm{cm}^{2} \text {, } \\
5 \mathrm{~min} . \\
\text { PU: } 1 \mathrm{MHz}, 2 \mathrm{~W} / \mathrm{cm}^{2} \text {, } \\
\text { 1:4; } 5 \text { minutes. } 10 \\
\text { sessions. }\end{array}$ & $\begin{array}{l}\text { VAS, WOMAC, } \\
\text { WT } 20 \\
\text { minutes, } \\
\text { goniometer }\end{array}$ & $\begin{array}{l}\text { Pain, } \\
\text { stiffness, } \\
\text { physical } \\
\text { function, } \\
\text { ROM, } \\
\text { functional } \\
\text { performance. }\end{array}$ & $\begin{array}{l}\text { All, } \uparrow \text { pain, stiffness } \\
\text { and physical } \\
\text { function, } \\
\text { PU } \uparrow \uparrow .\end{array}$ & 8 \\
\hline $\begin{array}{l}\text { Mao-Hsiung } \\
\text { Huang et al. } \\
(2005)(14)\end{array}$ & 120 & $\begin{array}{l}\text { PE } \times C U+ \\
P E \times P U+ \\
P E \times C G\end{array}$ & $\begin{array}{l}\mathrm{CU}: 1 \mathrm{MHz} \text { e } 1.5 \mathrm{~W} / \\
\mathrm{cm}^{2}, 5 \text { minutes per } \\
\text { each spot, totalizing } \\
25 \mathrm{~cm}^{2} \text {. } \\
\mathrm{PU}: 1 \mathrm{MHz} \text { e } 2.5 \mathrm{~W} / \\
\mathrm{cm}^{2}, 1: 4,5 \text { minutes } \\
\text { per each spot, } \\
\text { totalizing } 25 \mathrm{~cm}^{2} \text {. } \\
24 \text { sessions. }\end{array}$ & $\begin{array}{l}\text { VAS, } \\
\text { Lequesne, WT } \\
50 \text { minutes, } \\
\text { goniometer, } \\
\text { dynamometry. }\end{array}$ & $\begin{array}{l}\text { Pain, } \\
\text { discomfort, } \\
\text { physical } \\
\text { function, } \\
\text { MS, ROM, } \\
\text { functional } \\
\text { performance. }\end{array}$ & $\begin{array}{l}\text { All, except o CG, } \uparrow \\
\text { pain, discomfort and } \\
\text { muscle strenght, } \\
\text { with PU } \uparrow \uparrow . \\
\text { CU e PU } \uparrow \text { walking } \\
\text { speed e ROM. } \\
\text { PU } \uparrow \uparrow \text { walking } \\
\text { speed. }\end{array}$ & 5 \\
\hline $\begin{array}{l}\text { Huang et al. } \\
\text { (2005) (15) }\end{array}$ & 140 & $\begin{array}{l}P E \times P U+P E \\
\times P U+P E+ \\
H A \times C G\end{array}$ & $\begin{array}{l}\text { PU: } 1 \mathrm{MHz}, 2.5 \mathrm{~W} / \\
\mathrm{cm}^{2}, 1: 4 \text { per } 5 \\
\text { minutes for each } \\
\text { treated spot, on } \\
\text { total of } 25 \mathrm{~cm}^{2} .24 \\
\text { sessions. }\end{array}$ & $\begin{array}{l}\text { VAS, } \\
\text { Lequesne, } \\
\text { WT } 50 \\
\text { minutes, } \\
\text { goniometer, } \\
\text { dynamometry. }\end{array}$ & $\begin{array}{l}\text { Pain, } \\
\text { discomfort, } \\
\text { physical } \\
\text { function, } \\
\text { ROM, MS, } \\
\text { functional } \\
\text { performance. }\end{array}$ & $\begin{array}{l}\text { All, except CG, } \\
\uparrow \mathrm{MS} \text {, pain and } \\
\text { discomfort. Groups } \\
\text { with PU } \uparrow \text { ROM } \\
\text { and functional } \\
\text { performance. } \\
\mathrm{PE}+\mathrm{PU}+\mathrm{HA} \\
\uparrow \text { functional } \\
\text { performance. }\end{array}$ & 7 \\
\hline $\begin{array}{l}\text { Cakir et al. } \\
\text { (2013) (16) }\end{array}$ & 58 & $\begin{array}{l}P E+C U x \\
P E+P U x \\
P E+\text { placebo }\end{array}$ & $\begin{array}{l}\mathrm{CU}: 1 \mathrm{MHz} \text { e } 1 \mathrm{~W} / \\
\mathrm{cm}^{2}, 12 \text { minutes. } \\
\text { PU: } 1 \mathrm{MHz}, 1 \mathrm{~W} / \mathrm{cm}^{2} \text {, } \\
\text { 1:4, } 12 \text { minutes } 10 \\
\text { sessions. }\end{array}$ & $\begin{array}{l}\text { VAS, WOMAC, } \\
\text { WT } 20 \\
\text { minutes. }\end{array}$ & $\begin{array}{l}\text { Pain, } \\
\text { stiffness, } \\
\text { physical } \\
\text { function, } \\
\text { functional } \\
\text { performance. }\end{array}$ & All $\uparrow$ & 7 \\
\hline \multicolumn{8}{|c|}{ Therapeutic continous ultrasound: } \\
\hline $\begin{array}{l}\text { Mascarin et } \\
\text { al. (2012) (5) }\end{array}$ & 40 & $\begin{array}{l}\text { TENS } \times \text { CU } \\
\times \mathrm{PE}\end{array}$ & $\begin{array}{l}\text { TENS: } 100 \mathrm{~Hz} \text { e } 50 \\
\mu \mathrm{s} \text {, int till the sensory } \\
\text { threshold, } 20 \text { minutes } \\
\mathrm{CU}: 1 \mathrm{MHz}, 0.8 \mathrm{~W} / \\
\mathrm{cm}^{2}, 3 \text { a } 4 \text { minutes } \\
24 \text { sessions. }\end{array}$ & $\begin{array}{l}\text { VAS, WOMAC, } \\
\text { goniometer, } \\
\text { WT6'. }\end{array}$ & $\begin{array}{l}\text { Pain, physical } \\
\text { function, } \\
\text { functional } \\
\text { performance, } \\
\text { ROM. }\end{array}$ & $\begin{array}{l}\text { PE e CU } \uparrow \text { functional } \\
\text { performance. } \\
\text { All } \uparrow \text { pain e physical } \\
\text { function. }\end{array}$ & 6 \\
\hline $\begin{array}{l}\text { Ozgonenel } \\
\text { et al. (2008) } \\
\text { (17) }\end{array}$ & 67 & CU x placebo & $\begin{array}{l}\mathrm{CU}: 1 \mathrm{MHz} / 1 \mathrm{~W} / \mathrm{cm}^{2} \text {, } \\
5 \text { minutes } \\
10 \text { sessions. }\end{array}$ & $\begin{array}{l}\text { VAS, WOMAC, } \\
\text { WT } \\
50 \text { minutes. }\end{array}$ & $\begin{array}{l}\text { Pain, physical } \\
\text { function, } \\
\text { stiffness, } \\
\text { functional } \\
\text { performance. }\end{array}$ & $\mathrm{CU} \uparrow$ & 7 \\
\hline $\begin{array}{l}\text { Luksurapan } \\
\text { et al. (2013) } \\
\text { (18) }\end{array}$ & 46 & $\mathrm{CU} \times \mathrm{PP}$ & $\begin{array}{l}\mathrm{CU}: 1 \mathrm{MHz} \text { e } 1 \mathrm{~W} / \mathrm{cm}^{2} \text {, } \\
10 \text { minutes. } \\
\text { PP: } 1 \mathrm{MHz} \text { e } 1 \mathrm{~W} / \\
\mathrm{cm}^{2}, 10 \text { minutes } \\
+ \text { Piroxicam. } 10 \\
\text { sessions. }\end{array}$ & VAS, WOMAC. & $\begin{array}{l}\text { Pain, } \\
\text { stiffness, } \\
\text { physical } \\
\text { function. }\end{array}$ & $\begin{array}{l}\text { Both } \uparrow, P P \uparrow \uparrow, \text { but } \\
\text { without significant } \\
\text { difference. }\end{array}$ & 10 \\
\hline
\end{tabular}


Ferronato L, Cunha HM, Machado PM, Souza GS, Limana MD, Avelar NCP.

\begin{tabular}{|c|c|c|c|c|c|c|c|}
\hline Study & Sample & Groups & Program & Instruments & $\begin{array}{l}\text { Endpoints } \\
\text { Evaluated }\end{array}$ & Results & PEDro \\
\hline $\begin{array}{l}\text { Kulcu et al. } \\
\text { (2009) (19) }\end{array}$ & 45 & $\begin{array}{l}\text { PEF x CU } x \\
\text { CG }\end{array}$ & $\begin{array}{l}\text { PEF: } 2 \mathrm{~Hz}, 100 \mathrm{~Hz}, 25 \\
\mathrm{~Hz} \text {, consecutively, } 35 \\
\text { minutes. } \\
\mathrm{CU}: 1 \mathrm{MHz}, 1.5 \mathrm{~W} / \\
\mathrm{cm} 2 \text {, } \\
10 \text { minutes. }\end{array}$ & VAS, WOMAC. & $\begin{array}{l}\text { Pain, } \\
\text { stiffness, } \\
\text { physical } \\
\text { function. }\end{array}$ & PEF e CU $\uparrow$. & 5 \\
\hline $\begin{array}{l}\text { Özgüçlü et } \\
\text { al.(2010) } \\
(20)\end{array}$ & 40 & $\begin{array}{l}\mathrm{PEF}+\mathrm{HC} \\
+\mathrm{CU}+\mathrm{PE} \\
\times \mathrm{HC}+\mathrm{CU} \\
+\mathrm{PE}\end{array}$ & $\begin{array}{l}\text { PEF: } 50 \mathrm{~Hz} \text {; } 30-\mathrm{G} \text {; } \\
90 \text { seconds break, } \\
30 \text { minutes } \mathrm{HC}: 20 \\
\text { minutes } \\
\mathrm{CU}: 1 \mathrm{MHz} \text { e } 1.5 \mathrm{~W} / \\
\mathrm{cm}^{2} \text {, } \\
5 \text { minutes } \\
10 \text { sessions. }\end{array}$ & VAS, WOMAC. & $\begin{array}{l}\text { Pain, } \\
\text { stiffness, } \\
\text { physical } \\
\text { function. }\end{array}$ & Both $\uparrow$ & 6 \\
\hline $\begin{array}{l}\text { Ulus et al. } \\
\text { (2012) (21) }\end{array}$ & 42 & $\begin{array}{l}\mathrm{CU}+\mathrm{HC} \\
+\mathrm{IC}+\mathrm{PE} \\
\times \text { Placebo } \\
+\mathrm{HC}+\mathrm{IC} \\
+\mathrm{PE}\end{array}$ & $\begin{array}{l}\text { CU: } 1 \mathrm{MHz} \text { e } 1 \mathrm{~W} / \mathrm{cm}^{2} \text {, } \\
10 \text { minutes } \\
\mathrm{HC}: 20 \text { minutes } \\
\text { IC: } 10 \text { minutes } \\
15 \text { sessions. }\end{array}$ & $\begin{array}{l}\text { VAS, WOMAC, } \\
\text { WT } 50 \\
\text { minutes, } \\
\text { Lequesne, } \\
\text { HADS. }\end{array}$ & $\begin{array}{l}\text { Pain, physical } \\
\text { function, } \\
\text { stiffness, } \\
\text { functional } \\
\text { performance, } \\
\text { discomfort, } \\
\text { psychological } \\
\text { state. }\end{array}$ & Both $\uparrow$ & 7 \\
\hline $\begin{array}{l}\text { Bennell et al. } \\
(2005)(22)\end{array}$ & 119 & MT x Placebo & $\begin{array}{l}\text { MT: } 12 \text { weeks of } \\
\text { service }+12 \text { weeks } \\
\text { of self-management. }\end{array}$ & $\begin{array}{l}\text { VAS, WOMAC, } \\
\text { Likert scale, } \\
\text { SF-36, AQol, } \\
\text { dynamometry, } \\
\text { step test for } \\
\text { balance. }\end{array}$ & $\begin{array}{l}\text { Pain, physical } \\
\text { function, } \\
\text { stiffness, } \\
\text { patient's } \\
\text { global } \\
\text { change, life } \\
\text { quality, MS, } \\
\text { body balance. }\end{array}$ & $\begin{array}{l}\text { Both } \uparrow \text { pain and } \\
\text { patient's global } \\
\text { change. }\end{array}$ & 8 \\
\hline \multicolumn{8}{|c|}{ Neuromuscular Electrical Stimulation: } \\
\hline $\begin{array}{l}\text { Imoto et al. } \\
(2013)(6)\end{array}$ & 82 & $\begin{array}{l}\text { NMEE + PE } \\
\times \text { CG }\end{array}$ & $\begin{array}{l}\text { NMEE: } 50 \mathrm{~Hz}, 250 \mu \mathrm{s} \text {, } \\
\text { maximum tolerated } \\
\text { intensity, TON: } \\
\text { 10s, TOFF: } 30 \mathrm{~s}, 20 \\
\text { minutes }\end{array}$ & $\begin{array}{l}\text { VAS, TUG, } \\
\text { Lequesne, } \\
\text { DAL scale. }\end{array}$ & $\begin{array}{l}\text { Pain, physical } \\
\text { function, } \\
\text { functional } \\
\text { performance, } \\
\text { discomfort, } \\
\text { DAL. }\end{array}$ & $\begin{array}{l}\text { NMEE } \uparrow \text { pain, } \\
\text { physical function, } \\
\text { discomfort and DAL. }\end{array}$ & 7 \\
\hline $\begin{array}{l}\text { Vaz et al. } \\
\text { (2013) (23) }\end{array}$ & 12 & NMEE & $\begin{array}{l}\text { NMEE: } 80 \mathrm{~Hz} \text {, } \\
400 \mathrm{~ms} \text {, maximum } \\
\text { intensity tolerated. } 24 \\
\text { sessions. }\end{array}$ & $\begin{array}{l}\text { WOMAC, } \\
\text { ultrasonography, } \\
\text { dynamometry. }\end{array}$ & $\begin{array}{l}\text { Pain, } \\
\text { stiffness, } \\
\text { physical } \\
\text { function, } \\
\text { muscle } \\
\text { structure, } \\
\text { MS. }\end{array}$ & $\begin{array}{l}\text { NMEE } \uparrow \text { muscle } \\
\text { thickness increase } \\
\text { and fascicle length, } \\
\text { MS, pain, stiffness } \\
\text { and physical } \\
\text { function. }\end{array}$ & - \\
\hline $\begin{array}{l}\text { Elboim- } \\
\text { Gabyzon et } \\
\text { al. (2013) } \\
\text { (24) }\end{array}$ & 50 & $\begin{array}{l}\text { NMEE + PE } \\
x \text { PE }\end{array}$ & $\begin{array}{l}\text { NMEE: } 75 \mathrm{~Hz}, 250 \mu \mathrm{s} \text {, } \\
\text { maximum tolerated } \\
\text { intensity, TON: } \\
\text { 10s, TOFF: } 50 \mathrm{~s}, 45 \\
\text { minutes } 12 \text { sessions. }\end{array}$ & $\begin{array}{l}\text { VAS, WOMAC, } \\
\text { WT10m, } \\
\text { TUG, U'nDT, } \\
\text { myometry. }\end{array}$ & $\begin{array}{l}\text { Pain, } \\
\text { stiffness, } \\
\text { physical } \\
\text { function, } \\
\text { functional } \\
\text { performance, } \\
\text { muscle } \\
\text { performance. }\end{array}$ & $\begin{array}{l}\text { NMEE }+\mathrm{PE} \uparrow \\
\text { pain and muscle } \\
\text { activation. }\end{array}$ & 5 \\
\hline
\end{tabular}




\begin{tabular}{|c|c|c|c|c|c|c|c|}
\hline Study & Sample & Groups & Program & Instruments & $\begin{array}{l}\text { Endpoints } \\
\text { Evaluated }\end{array}$ & Results & PEDro \\
\hline $\begin{array}{l}\text { Durmus et al. } \\
\text { (2007) (25) }\end{array}$ & 50 & $\begin{array}{l}\text { NMEE x } \\
\text { Biofeedback } \\
+ \text { PE }\end{array}$ & $\begin{array}{l}\text { NMEE: } 50 \mathrm{~Hz}, 200 \mu \mathrm{s} \text {, } \\
\text { visible contraction } \\
\text { intensity, TON: } \\
\text { 10s, TOFF: } 10 \mathrm{~s}, 20 \\
\text { minutes } 20 \text { sessions. }\end{array}$ & $\begin{array}{l}\text { VAS, WOMAC, } \\
\text { MR test, } 10 \\
\text { MR, } \\
\text { WT } 50 \\
\text { minutes, } \\
\text { U'nDT. }\end{array}$ & $\begin{array}{l}\text { Pain, } \\
\text { stiffness, } \\
\text { physical } \\
\text { function, } \\
\text { functional } \\
\text { performance, } \\
\text { MS. }\end{array}$ & Both $\uparrow$ & 5 \\
\hline $\begin{array}{l}\text { Gaines et al. } \\
\text { (2004) (26) }\end{array}$ & 38 & NMEE x CG & $\begin{array}{l}\text { NMEE: visible } \\
\text { contraction intensity, } \\
\text { TON: } 10 \text { s, TOFF: } 50 \text { s, } \\
15 \text { minutes. }\end{array}$ & $\begin{array}{l}\text { Pain diary, } \\
\text { McGill pain } \\
\text { quiz, } \\
\text { AIMS2-PS. }\end{array}$ & Pain. & $\begin{array}{l}\text { NMEE } \uparrow \text { pain only } \\
\text { after } 15 \text { minutes. }\end{array}$ & 5 \\
\hline $\begin{array}{l}\text { Bruce-Brand } \\
\text { et al.(2012) } \\
\text { (27) }\end{array}$ & 41 & $\begin{array}{l}\text { PE x NMEE } \\
\times C G\end{array}$ & $\begin{array}{l}\text { NMEE: } 50 \mathrm{~Hz}, 100- \\
400 \mu \mathrm{s} \text {, maximum } \\
\text { tolerated intensity, } \\
\text { TON: } 10 \mathrm{~s} \text {, TOFF: } 50 \mathrm{~s} \text {, } \\
20 \text { minutes. }\end{array}$ & $\begin{array}{l}\text { WOMAC, WT } \\
25 \text { minutes, } \\
\text { GUSDT, } \\
\text { U'nDT, SF-36, } \\
\text { dynamometry, } \\
\text { magnetic } \\
\text { resonance. }\end{array}$ & $\begin{array}{l}\text { Pain, } \\
\text { stiffness, } \\
\text { physical } \\
\text { function, } \\
\text { functional } \\
\text { performance, } \\
\text { life quality, } \\
\text { muscle } \\
\text { performance. }\end{array}$ & $\begin{array}{l}\text { NMEE e PE } \\
\uparrow \text { functional } \\
\text { performance and } \\
\text { increased cross- } \\
\text { sectional area of the } \\
\text { quadriceps. }\end{array}$ & 5 \\
\hline $\begin{array}{l}\text { Burch et al. } \\
\text { (2008) (28) }\end{array}$ & 116 & $\begin{array}{l}\text { IC }+ \\
\text { standardized } \\
\text { muscle } \\
\text { stimulation } \\
\text { x TENS }\end{array}$ & $\begin{array}{l}\text { IC: } 5.000 \mathrm{~Hz}, 1 \mathrm{e} \\
150 \mathrm{~Hz} \text {, mild tingling } \\
\text { intensity, } 15 \text { minutes } \\
\text { standardized muscle } \\
\text { stimulation: } 50 \mathrm{~Hz} \text { for } \\
200 \mathrm{~ms} \text { each } 1500 \mathrm{~ms} \text {, } \\
\text { intensity between } \\
3.39 \mu \mathrm{s} \text { e } 102.2 \mu \mathrm{s}, \\
\text { with average of } 16.26 \\
\mathrm{~mA}, 20 \text { minutes } \\
\text { TENS: } 0.2 \mathrm{~Hz}, 300 \mu \mathrm{s} \text {, } \\
0.5 \mathrm{~mA} \text { of intensity, } 35 \\
\text { minutes }\end{array}$ & VAS, WOMAC. & $\begin{array}{l}\text { Pain, } \\
\text { stiffness, } \\
\text { physical } \\
\text { function, life } \\
\text { quality. }\end{array}$ & $\begin{array}{l}\text { IC }+ \text { standarized } \\
\text { muscle stimulation } \uparrow\end{array}$ & 5 \\
\hline $\begin{array}{l}\text { Palmieri- } \\
\text { Smith et al. } \\
\text { (2010) (29) }\end{array}$ & 30 & NMEE x CG & $\begin{array}{l}\text { NMEE: } 2.500 \mathrm{~Hz}, 50 \\
\text { bursts/s, maximum } \\
\text { tolerated intensity, } \\
\text { TON: } 10 \text { s, TOFF: } 50 \mathrm{~s} \text {, } \\
10 \text { contractions. } 12 \\
\text { sessions. }\end{array}$ & $\begin{array}{l}\text { WOMAC, } \\
\text { dynamometry, } \\
\text { WT 12-19 } \\
\text { minutes. }\end{array}$ & $\begin{array}{l}\text { Pain, } \\
\text { stiffness, } \\
\text { physical } \\
\text { function, MS } \\
\text { and muscle } \\
\text { activation, } \\
\text { functional } \\
\text { performance. }\end{array}$ & $\leftrightarrow$ & 7 \\
\hline \multicolumn{8}{|c|}{ Transcutaneous electrical nerve stimulation: } \\
\hline $\begin{array}{l}\text { Law et al. } \\
(2004)(30)\end{array}$ & 34 & $\begin{array}{l}\text { multiple } \\
\text { frequencies } \\
\text { TENS } x \\
\text { placebo }\end{array}$ & $\begin{array}{l}\text { TENS: } 25-35 \mathrm{~mA} \text { of } \\
\text { intensity, Frequency: } \\
2 \mathrm{~Hz} \text { ou } 100 \mathrm{~Hz} \text { or } \\
2 / 100 \mathrm{~Hz} \text { alternated; } \\
576 \mu \mathrm{s} \text { or } 200 \mu \mathrm{s} \\
\text { or } 576 / 200 \mu \mathrm{s}, \\
\text { respectively, } 40 \\
\text { minutes } 10 \text { sessions. }\end{array}$ & $\begin{array}{l}\text { VAS, } \\
\text { goniometer, } \\
\text { TUG. }\end{array}$ & $\begin{array}{l}\text { Pain, ROM, } \\
\text { functional } \\
\text { performance. }\end{array}$ & $\begin{array}{l}\text { All, except placebo, } \\
\uparrow\end{array}$ & 7 \\
\hline
\end{tabular}


Ferronato L, Cunha HM, Machado PM, Souza GS, Limana MD, Avelar NCP.

\begin{tabular}{|c|c|c|c|c|c|c|c|}
\hline Study & Sample & Groups & Program & Instruments & $\begin{array}{l}\text { Endpoints } \\
\text { Evaluated }\end{array}$ & Results & PEDro \\
\hline $\begin{array}{l}\text { Atamaz et al. } \\
\text { (2012) (31) }\end{array}$ & 175 & $\begin{array}{l}\text { TENS + PE } \\
x I C+P E x \\
\text { SWD + PE } \\
x \text { placebos } \\
+P E\end{array}$ & $\begin{array}{l}\text { TENS: } 80 \mathrm{~Hz}, 10- \\
30 \mathrm{~mA} \text { intensity. } \\
\text { IC: } 100 \mathrm{~Hz}, 4 \mathrm{KHz} \text {, } \\
\text { tactile sensation } \\
\text { intensity. } \\
\text { SWD: } 27.12 \mathrm{MHz} \text {, } \\
300 \mathrm{~W} \text { input and } \\
\text { average of } 3.2 \mathrm{~W} .15 \\
\text { sessions. }\end{array}$ & $\begin{array}{l}\text { VAS, WOMAC, } \\
\text { goniometer, } \\
\text { WT15m, } \\
\text { Nottingham } \\
\text { Health Profile. }\end{array}$ & $\begin{array}{l}\text { Pain, } \\
\text { stiffness, } \\
\text { physical } \\
\text { function, } \\
\text { ROM, } \\
\text { functional } \\
\text { performance, } \\
\text { life quality. }\end{array}$ & $\begin{array}{l}\text { TENS, IC, active } \\
\text { SWD, lower intake } \\
\text { of paracetamol. }\end{array}$ & 9 \\
\hline $\begin{array}{l}\text { Cetin et al. } \\
(2008)(32)\end{array}$ & 100 & $\begin{array}{l}\text { SWD + HC } \\
+\mathrm{PE} \times \mathrm{TENS} \\
+\mathrm{HC}+\mathrm{PE} \times \\
\mathrm{CU}+\mathrm{HC}+ \\
\mathrm{PE} \times \mathrm{HC}+\mathrm{PE} \\
\times \mathrm{PE}\end{array}$ & $\begin{array}{l}\text { SWD: I: } 27.12 \mathrm{MHz} \text {, } \\
15 \text { minutes } \\
\text { TENS: } 60-110 \mathrm{~Hz}, 60 \\
\mu \mathrm{s}, \text { no contraction } \\
\text { maximum intensity, } \\
20 \text { minutes. } \\
\mathrm{CU}: 1 \mathrm{MHz}, 1.5 \mathrm{~W} / \\
\mathrm{cm}^{2}, 10 \text { minutes. }\end{array}$ & $\begin{array}{l}\text { VAS, } \\
\text { Lequesne, } \\
\text { ISKOA, WT } \\
50 \text { minutes, } \\
\text { dynamometry. }\end{array}$ & $\begin{array}{l}\text { Pain, } \\
\text { discomfort, } \\
\text { physical } \\
\text { function, } \\
\text { functional } \\
\text { performance, } \\
\text { MS. }\end{array}$ & $\begin{array}{l}\text { All } \uparrow \text { pain and } \\
\text { incapacity (ISKOA). } \\
\text { All, except isolated } \\
\mathrm{PE} \text {, } \uparrow \text { pain, } \\
\text { discomfort, physical } \\
\text { function e MS. } \\
\text { SWD e TENS } \uparrow \uparrow\end{array}$ & 6 \\
\hline $\begin{array}{l}\text { Cheing et al. } \\
\text { (2004) (33) }\end{array}$ & 62 & $\begin{array}{l}\text { TENS } x \\
\text { placebo x PE } \\
x \text { TENS + PE }\end{array}$ & $\begin{array}{l}\text { TENS: } 80 \mathrm{~Hz}, 140 \mu \mathrm{s} \text {, } \\
\text { tingling intensity, } 60 \\
\text { minutes, } \\
20 \text { sessions. }\end{array}$ & $\begin{array}{l}\text { Dynamometry, } \\
\text { spatiotemporal } \\
\text { parameters } \\
\text { of the march, } \\
\text { goniometer. }\end{array}$ & $\begin{array}{l}\text { Muscle } \\
\text { performance, } \\
\text { ROM. }\end{array}$ & $\begin{array}{l}\text { TENS + PE shows } \\
\text { a trend in the } \\
\text { improvement of } \\
\text { physical parameters, } \\
\text { but showed } \\
\text { no significant } \\
\text { difference. }\end{array}$ & 5 \\
\hline $\begin{array}{l}\text { Pietrosimone } \\
\text { et al. (2011) } \\
\text { (34) }\end{array}$ & 36 & $\begin{array}{l}\text { TENS + PE x } \\
\text { placebo + PE } \\
x \text { PE }\end{array}$ & $\begin{array}{l}\text { TENS: } 150 \mathrm{~Hz} \text {, } \\
150 \mu \mathrm{s}, \text { strong } \\
\text { sensorial intensity, for } \\
\text { at least } 8 \text { hrs a day, } \\
12 \text { sessions. }\end{array}$ & $\begin{array}{l}\text { WOMAC, } \\
\text { dynamometry. }\end{array}$ & $\begin{array}{l}\text { Pain, } \\
\text { stiffness, } \\
\text { physical } \\
\text { function, } \\
\text { muscle } \\
\text { activation. }\end{array}$ & $\begin{array}{l}\text { TENS + PE } \uparrow \\
\text { muscle activation. } \\
\text { All } \uparrow \text { pain, stiffness e } \\
\text { physical function. }\end{array}$ & 7 \\
\hline $\begin{array}{l}\text { Kolen et al. } \\
\text { (2012) (35) }\end{array}$ & 74 & $\begin{array}{l}\text { TENS on } \\
\text { different } \\
\text { spots. }\end{array}$ & $\begin{array}{l}\text { TENS: } 80 \mathrm{~Hz}, 100 \mu \mathrm{s} \text {, } \\
\text { maximum tolerated } \\
\text { intensity, } 30-45 \\
\text { minutes. }\end{array}$ & $\begin{array}{l}\text { VAS, WOMAC, } \\
\text { WT6', } \\
\text { dynamometry, } \\
\text { goniometer, } \\
\text { HADS, Pain } \\
\text { Anxiety } \\
\text { Symptoms } \\
\text { Scale, Pain } \\
\text { Catastrophizing } \\
\text { Scale, } \\
\text { satisfaction } \\
\text { quiz. }\end{array}$ & $\begin{array}{l}\text { Pain, } \\
\text { stiffness, } \\
\text { physical } \\
\text { function, } \\
\text { functional } \\
\text { performance, } \\
\text { muscle } \\
\text { performance, } \\
\text { ROM. }\end{array}$ & $\begin{array}{l}\text { TENS } \uparrow \text { pain. } \\
\uparrow \uparrow \text { when applied on } \\
\text { low skin resistance } \\
\text { spots. }\end{array}$ & 7 \\
\hline $\begin{array}{l}\text { Selfe et al. } \\
(2008)(36)\end{array}$ & 37 & $\begin{array}{l}\text { NIN x } \\
\text { placebo. }\end{array}$ & $\begin{array}{l}\text { TENS on low } \\
\text { resistance spots, 20- } \\
30 \text { minutes, } \\
17 \text { sessions. }\end{array}$ & $\begin{array}{l}\text { VAS, WOMAC, } \\
\text { SF-36. }\end{array}$ & $\begin{array}{l}\text { Pain, } \\
\text { stiffness, } \\
\text { physical } \\
\text { function, } \\
\text { global } \\
\text { evaluation, } \\
\text { life quality. }\end{array}$ & $\begin{array}{l}\text { NIN } \text { 个pain, without } \\
\text { meaningful } \\
\text { differences. NIN } \uparrow \\
\text { vitality (subscale } \\
\text { SF-36) and global } \\
\text { evaluation of the } \\
\text { patient. }\end{array}$ & 7 \\
\hline $\begin{array}{l}\text { Vance et al. } \\
(2012)(37)\end{array}$ & 75 & $\begin{array}{l}\text { High } \\
\text { frequencyTENS } \\
\text { x low } \\
\text { frequencyTENS } \\
\text { x placebo }\end{array}$ & $\begin{array}{l}\text { TENS: } 100 \mathrm{~Hz} \text { or } 4 \mathrm{~Hz} \text {, } \\
100 \mu \mathrm{s}, 10 \% \text { below } \\
\text { the motor threshold } \\
\text { intensity, } 40-50 \\
\text { minutes, } 1 \text { session. }\end{array}$ & $\begin{array}{l}\text { VAS, } \\
\text { quantitative } \\
\text { sensory } \\
\text { testing, TUG. }\end{array}$ & $\begin{array}{l}\text { Pain, } \\
\text { functional } \\
\text { performance. }\end{array}$ & $\begin{array}{l}\text { All, except placebo, } \\
\text { pressure pain. All } \uparrow \\
\text { rest pain TUG. }\end{array}$ & 8 \\
\hline
\end{tabular}




\begin{tabular}{|c|c|c|c|c|c|c|c|}
\hline Study & Sample & Groups & Program & Instruments & $\begin{array}{l}\text { Endpoints } \\
\text { Evaluated }\end{array}$ & Results & PEDro \\
\hline $\begin{array}{l}\text { Pietrosimone } \\
\text { et al. (2009) } \\
\text { (38) }\end{array}$ & 33 & $\begin{array}{l}\text { TENS } x \\
\text { criotera- } \\
\text { phy } x \text { CG }\end{array}$ & $\begin{array}{l}\text { TENS: } 150 \mathrm{~Hz}, 150 \mu \mathrm{s} \text {, } \\
\text { sensory intensity, } 45 \\
\text { minutes } \\
\text { Crioteraphy: } 2 \text { bags } \\
\text { of } 1.5 \mathrm{~L} \text { of ice, one on } \\
\text { the front and another } \\
\text { on the back of the } \\
\text { knee, } 20 \text { minutes } 1 \\
\text { session. }\end{array}$ & Dynamometry. & $\begin{array}{l}\text { Muscle } \\
\text { activation. }\end{array}$ & $\begin{array}{l}\text { All, except CG } \\
\uparrow \text { quadriceps } \\
\text { activation } \\
\text { immediately after } \\
\text { application. }\end{array}$ & 6 \\
\hline $\begin{array}{l}\text { Chen et al. } \\
\text { (2013) (39) }\end{array}$ & 50 & HA x TENS & $\begin{array}{l}\text { TENS: } 3 \mathrm{~Hz} \text { and } 20 \\
\mathrm{~Hz}, 200 \mu \mathrm{s} \text {, maximum } \\
\text { tolerated intensity, } 20 \\
\text { minutes } 12 \text { sessions. } \\
\mathrm{HA}: 2.5 \mathrm{ml}, 5 \text { shots. }\end{array}$ & $\begin{array}{l}\text { VAS, } \\
\text { Lequesne, } \\
\text { goniometer, } \\
\text { WT, pressure } \\
\text { algometry. }\end{array}$ & $\begin{array}{l}\text { Pain, physical } \\
\text { function, } \\
\text { discomfort, } \\
\text { ROM, } \\
\text { functional } \\
\text { performance, } \\
\text { global } \\
\text { evaluation, } \\
\text { DAL. }\end{array}$ & $\begin{array}{l}\text { TENS } \uparrow \text { pain, } \\
\text { discomfort and } \\
\text { physical function. }\end{array}$ & 6 \\
\hline $\begin{array}{l}\text { Paker et al. } \\
\text { (2006) (40) }\end{array}$ & 60 & HA x TENS & $\begin{array}{l}\text { TENS: } 150 \mathrm{~Hz}, 20 \\
\text { minutes per } 1 \text { hour. } \\
\text { HA: } 3 \text { shots of hylan } \\
\text { GF20. }\end{array}$ & $\begin{array}{l}\text { WOMAC, } \\
\text { Lequesne, } \\
\text { SF-36. }\end{array}$ & $\begin{array}{l}\text { Pain, } \\
\text { stiffness, } \\
\text { physical } \\
\text { function, } \\
\text { discomfort, } \\
\text { life quality. }\end{array}$ & $\begin{array}{l}\text { Both } \uparrow \text { pain, physical } \\
\text { function e stiffness. } \\
\text { HA } \uparrow \text { physical } \\
\text { function. }\end{array}$ & 6 \\
\hline $\begin{array}{l}\text { Pietrosimone } \\
\text { et al. (2010) } \\
\text { (41) }\end{array}$ & 36 & $\begin{array}{l}\text { TENS + PE X } \\
\text { placebo + PE } \\
x P E\end{array}$ & $\begin{array}{l}\text { TENS: } 150 \mathrm{~Hz}, 150 \mu \mathrm{s} \text {, } \\
\text { Strong intensity } \\
\text { without contraction } \\
\text { for at least } 8 \text { hours } \\
\text { a day. }\end{array}$ & $\begin{array}{l}\text { WOMAC, } \\
\text { dynamometry, } \\
\text { three- } \\
\text { dimensional } \\
\text { march } \\
\text { analysis at } 15 \\
\text { meters. }\end{array}$ & $\begin{array}{l}\text { Pain, } \\
\text { stiffness, } \\
\text { physical } \\
\text { function, } \\
\text { functional } \\
\text { performance, } \\
\text { muscle } \\
\text { activation, } \\
\text { MS. }\end{array}$ & $\begin{array}{l}\text { All } \uparrow \\
\leftrightarrow \text { knee flexion } \\
\text { angle. }\end{array}$ & 7 \\
\hline \multicolumn{8}{|c|}{ Pulsed electrical stimulation: } \\
\hline $\begin{array}{l}\text { Fary et al. } \\
\text { (2009) (42) }\end{array}$ & 3 & PES & $\begin{array}{l}\text { PES: } 100 \mathrm{~Hz}, 2 \mathrm{~ms} \text {, } \\
\text { subsensorial intensity, } \\
\text { for at least } 8 \text { hours } \\
\text { a day. }\end{array}$ & $\begin{array}{l}\text { VAS, Likert } \\
\text { scale, SF- } \\
36, \text { global } \\
\text { perceived } \\
\text { effect scale, } \\
\text { accelerometry. }\end{array}$ & $\begin{array}{l}\text { Pain, physical } \\
\text { function, } \\
\text { global } \\
\text { evaluation, } \\
\text { life quality, } \\
\text { exercise. }\end{array}$ & $\begin{array}{l}\text { PES } \uparrow \text { symptoms, } \\
\text { supported by up to } \\
16 \text { weeks. }\end{array}$ & - \\
\hline $\begin{array}{l}\text { Garland et al. } \\
\text { (2007) (43) }\end{array}$ & 58 & $\begin{array}{l}\text { PES } x \\
\text { placebo }\end{array}$ & $\begin{array}{l}\text { PES: } 100 \mathrm{~Hz} \text {, } \\
\text { subsensorial intensity, } \\
6 \text { hours or more. }\end{array}$ & VAS, WOMAC. & $\begin{array}{l}\text { Pain, } \\
\text { stiffness, } \\
\text { physical } \\
\text { function, } \\
\text { global } \\
\text { evaluation. }\end{array}$ & PES $\uparrow$ & 8 \\
\hline $\begin{array}{l}\text { Fary et al. } \\
\text { (2011) (44) }\end{array}$ & 70 & $\begin{array}{l}\text { PES } x \\
\text { placebo }\end{array}$ & $\begin{array}{l}\text { PES: } 100 \mathrm{~Hz}, 4 \mathrm{~ms}, 7 \\
\text { hours a day. }\end{array}$ & $\begin{array}{l}\text { VAS, WOMAC, } \\
\text { SF-36, health } \\
\text { survey, } \\
\text { Human } \\
\text { Activity Profile, } \\
\text { accelerometry, } \\
\text { perceived } \\
\text { global effect } \\
\text { scale. }\end{array}$ & $\begin{array}{l}\text { Pain, } \\
\text { stiffness, } \\
\text { physical } \\
\text { function, } \\
\text { life quality, } \\
\text { exercise. }\end{array}$ & Both $\uparrow$ & 10 \\
\hline
\end{tabular}




\begin{tabular}{|c|c|c|c|c|c|c|c|}
\hline Study & Sample & Groups & Program & Instruments & $\begin{array}{l}\text { Endpoints } \\
\text { Evaluated }\end{array}$ & Results & PEDro \\
\hline \multicolumn{8}{|c|}{ Low level laser therapy: } \\
\hline $\begin{array}{l}\text { Kheshie et al. } \\
\text { (2014) (45) }\end{array}$ & 53 & $\begin{array}{l}\text { High intensity } \\
A L+P E x \\
\text { low intensity } \\
A L+P E x \\
\text { placebo }+ \text { PE }\end{array}$ & $\begin{array}{l}\text { High intensity AL: } \\
\text { Total power of } 1250 \mathrm{~J} \text {, } \\
610 \text { to } 810 \mathrm{~mJ} / \mathrm{cm} 2 \\
\text { application. } \\
\text { Low intensity AL: } \\
\text { Total power of } 1250 \mathrm{~J} \text {, } \\
800 \mathrm{~mW}, 1 \mathrm{KHz}, 50 \\
\mathrm{~J} / \mathrm{cm}^{2} \text {. }\end{array}$ & VAS, WOMAC. & $\begin{array}{l}\text { Pain, physical } \\
\text { function. }\end{array}$ & $\begin{array}{l}\text { Both active } \uparrow \\
\text { High intensity } A L \uparrow \uparrow\end{array}$ & 7 \\
\hline $\begin{array}{l}\text { Alfredo et al. } \\
\text { (2011) (7) }\end{array}$ & 40 & $\begin{array}{l}\mathrm{AL}+\mathrm{PEx} \\
\text { placebo }+\mathrm{PE}\end{array}$ & $\begin{array}{l}\text { AL: } 904 \mathrm{~nm}, 700 \mathrm{~Hz}, \\
60 \mathrm{~mW} \text { average, } \\
20 \mathrm{~mW} \text { maximum, } 27 \\
\mathrm{~J}, 3 \mathrm{~J} / \text { point total dose, } \\
50 \mathrm{~s} .\end{array}$ & $\begin{array}{l}\text { VAS, WOMAC, } \\
\text { Lequesne, } \\
\text { goniometer, } \\
\text { dynamometry. }\end{array}$ & $\begin{array}{l}\text { Pain, physical } \\
\text { function, } \\
\text { ROM, MS, life } \\
\text { quality. }\end{array}$ & $\begin{array}{l}\mathrm{AL} \uparrow \text { pain, physical } \\
\text { function e ROM. }\end{array}$ & 8 \\
\hline $\begin{array}{l}\text { Alghadir et al. } \\
(2013)(46)\end{array}$ & 40 & AL x placebo & $\begin{array}{l}\mathrm{AL}: 850 \mathrm{~nm}, 50 \mathrm{~mW} \text {, } \\
6 \mathrm{~J} / \text { point, } 60 \mathrm{~s} / \text { point, } \\
\text { with } 48 \mathrm{~J} / \mathrm{cm}^{2} .8 \\
\text { sessions. }\end{array}$ & $\begin{array}{l}\text { VAS, WOMAC, } \\
\text { WT } 15 \\
\text { minutes. }\end{array}$ & $\begin{array}{l}\text { Pain, physical } \\
\text { function, } \\
\text { functional } \\
\text { performance. }\end{array}$ & $A L \uparrow$ & - \\
\hline $\begin{array}{l}\text { Hegedus et } \\
\text { al. (2009) } \\
\text { (47) }\end{array}$ & 27 & AL x placebo & $\begin{array}{l}\text { AL: } 830 \mathrm{~nm}, 50 \mathrm{~mW} \text {, } \\
6 \mathrm{~J} / \text { point, } 48 \mathrm{~J} / \mathrm{cm}^{2} \\
\text { dose. LP: } 0.5 \mathrm{~mW} .8 \\
\text { sessions. }\end{array}$ & $\begin{array}{l}\text { VAS, Ritchie } \\
\text { index, } \\
\text { goniometer, } \\
\text { thermography. }\end{array}$ & $\begin{array}{l}\text { Pain, ROM, } \\
\text { local micro- } \\
\text { circulation, } \\
\text { pressure } \\
\text { sensitivity. }\end{array}$ & $\mathrm{AL} \uparrow$ & 6 \\
\hline $\begin{array}{l}\text { Al Rashoud } \\
\text { et al. (2014) } \\
(48)\end{array}$ & 49 & $\begin{array}{l}\text { AL on } \\
\text { acupuncture } \\
\text { points }+ \text { PE x } \\
\text { placebo }\end{array}$ & $\begin{array}{l}\text { AL: } 830 \mathrm{~nm}, 30 \mathrm{~mW} \text {, } \\
6 \mathrm{~J} \text { total per session, } \\
1.2 \mathrm{~J} / \text { point, } 4 \mathrm{~J} / \mathrm{cm}^{2} \text {, } \\
5 \text { points, } 40 \text { s. } 9 \\
\text { sessions. }\end{array}$ & VAS, SKFS. & $\begin{array}{l}\text { Pain, physical } \\
\text { function. }\end{array}$ & $\mathrm{AL} \uparrow$ & 6 \\
\hline $\begin{array}{l}\text { Yurtkuran et } \\
\text { al. (2007) } \\
\text { (49) }\end{array}$ & 52 & $\begin{array}{l}\text { AL on } \\
\text { acupuncture } \\
\text { points } x \\
\text { placebo }\end{array}$ & $\begin{array}{l}\text { AL: } 904 \mathrm{~nm}, 4 \mathrm{~mW} \text {, } \\
0.48 \mathrm{~J} \text { dose, } 10 \mathrm{~mW} / \\
\mathrm{cm}^{2} \text { power density, } \\
\text { 120s/point. } 10 \\
\text { sessions. }\end{array}$ & $\begin{array}{l}\text { VAS, } \\
\text { WT50m, knee } \\
\text { circumferen- } \\
\text { ce, Medial } \\
\text { Tenderness } \\
\text { Score, } \\
\text { Nottingham } \\
\text { Health Profile, } \\
\text { WOMAC. }\end{array}$ & $\begin{array}{l}\text { Pain, } \\
\text { functional } \\
\text { performance, } \\
\text { edema, knee } \\
\text { sensibility, } \\
\text { life quality, } \\
\text { stiffness, } \\
\text { physical } \\
\text { function. }\end{array}$ & $\begin{array}{l}\mathrm{AL} \uparrow \text { edema } \\
\text { reduction. }\end{array}$ & 8 \\
\hline $\begin{array}{l}\text { Tascioglu } \\
\text { et al.(2004) } \\
\text { (50) }\end{array}$ & 60 & $\begin{array}{l}3 \mathrm{~J} A L \times 1.5 \mathrm{~J} \\
\mathrm{AL} \times \mathrm{p} \text { placebo }\end{array}$ & $\begin{array}{l}\text { AL 830nm, 50mW. } 10 \\
\text { sessions. }\end{array}$ & VAS, WOMAC. & $\begin{array}{l}\text { Pain, } \\
\text { stiffness, } \\
\text { physical } \\
\text { function. }\end{array}$ & $\leftrightarrow$ & 5 \\
\hline
\end{tabular}

Note: CU: continous ultrasound; PU: pulsed ultrasound; ROM: range of motion; VAS: visual analog scale; WOMAC: Western Ontario and McMaster Universities Osteoarthritis; WT: walking test; PE: physical exercise; CG: control group; MS: muscle strenght; HA: hialuronic acid; SWD: shortwave diathermy; HC: hot compresses; TENS: Transcutaneous electrical nerve stimulation; ISKOA: index of severity for knee osteoarthritis; WT6': walking testo f 6 minutes; PP: piroxicam phonophoresis; PEF: pulsed eletromagnetic field; HADS: Hospital Anxiety and Depression Scale; MT: multimodality therapy; SF-36: life quality quiz; NMEE: Neuromuscular Electrical Stimulation; DAL: daily life activities; TUG: timed get up and go; U'NDT: up and downstairs test; MR: maximum resistance; AIMS2-PS: arthritis impact measurement scale 2-pain subscale; GUSDT: get up and sit down test; IC: intensityerferential current; NIN: Noninvasive intensityeractive neurostimulation; PES: pulsed eletric stimulation; AL: active laser; SKFS: saudi knee function scale; TO: time on; TOFF: time off.

$\uparrow$ : Effective $\leftrightarrow$ : Ineffective $\uparrow \uparrow:$ Greater efficacy 


\section{Discussion}

\section{Therapeutic Ultrasound}

Studies comparing pulsed and continuous ultrasound application effectiveness on pain, physical function stiffness, discomfort, range of motion (ROM), functional performance and muscle strength (FM), demonstrated that both application forms can be effective for patients with knee osteoarthritis. However, the increase in the variables analyzed was better in the group receiving the application of pulsed ultrasound $(13,14)$.

Huang et al. (15) checked the efficacy of exercise performed alone, pulsed ultrasound + exercise and pulsed ultrasound + exercise + hyaluronic acid in pain variables, discomfort and fibromyalgia. It was found that the groups with pulsed US were effective to increase the range of motion and functional performance and hyaluronic acid group was more effective in functional performance, discomfort and fibromyalgia. The parameters used in these studies (13-15) varied as: continous US ( $1 \mathrm{MHz}, 1.5$ to $2 \mathrm{~W} / \mathrm{cm}^{2}$, 5 minutes) and pulsed US ( $1 \mathrm{MHz}, 2$ to $2.5 \mathrm{~W} / \mathrm{cm}^{2}$ working cycle 1: 4, 5 minutes) with treatment time from 10 to 24 sessions.

Discordant results were obtained in the study of Cakir et al. (16), who reported improvement in pain, physical function, stiffness and functional performance, at the continuous US $\left(1 \mathrm{MHz}\right.$ and $1 \mathrm{~W} / \mathrm{cm}^{2}$, 12 minutes), pulsed US ( $1 \mathrm{MHz}, 1 \mathrm{~W} / \mathrm{cm}^{2}$ and a working cycle 1: 4, 12 minutes) and placebo US groups, with no differences among the groups, after 10 therapy sessions. All groups performed home exercises and the authors suggest that these exercises masked the US effects.

Mascarin et al. (5) have proven the effectiveness of 24 sessions with transcutaneous electrical neurostimulation therapy $(100 \mathrm{~Hz}$ and $50 \mu$ s, sensitive threshold intensity, 20 minutes), continuous US (1 MHz, 0.8 W / $\mathrm{cm}^{2}, 3-4$ minutes) and exercise in pain and physical function. The presented results demonstrated efficacy only of continuous US and exercise in functional performance. Concordant to this study, Ozgonenel et al. (17) observed the effectiveness of continued US ( $1 \mathrm{MHz}$ and $1 \mathrm{~W} / \mathrm{cm}^{2}, 5$ to 10 minutes) in pain, physical function, stiffness and functional performance for 10 sessions compared to the placebo group. Luksurapan et al. (18) found no significant difference between the Piroxicam phonophoresis and continuous US, sbecause both treatments showed improvements.

Kulcu et al. (19) verified the effectiveness of pulsed electromagnetic field therapy (PEMF) (frequency: $2 \mathrm{~Hz}, 100 \mathrm{~Hz}, 25 \mathrm{~Hz}$, consecutively, 35 minutes / session) and continued US ( $1 \mathrm{MHz}, 1.5 \mathrm{~W} / \mathrm{cm}^{2}, 10 \mathrm{~min}-$ utes) in pain, stiffness and physical function. Both therapies were effective for the analyzed variables. In contrast, Özgüçlü et al. (20) found no additional effects of pulsed electromagnetic field therapy $(50 \mathrm{~Hz}$, with an intensity of 30-G in a 90s interval, $30 \mathrm{~min}$ utes) to treatment with continuous US $(1 \mathrm{MHz}$ and $1.5 \mathrm{~W} / \mathrm{cm}^{2}, 5$ minutes), hot bag and exercise, with the same pain improvement, physical function and stiffness in treatments without pulsed eletromagnetic field for 10 sessions.

Discordant results were described by Ulus et al. (21) who verified efficiency of both the continuous US ( $1 \mathrm{MHz}$ and $1 \mathrm{~W} / \mathrm{cm}^{2}$ for 10 minutes) and the placebo US associated with interferential current (IF), hot packs and exercise, in pain, physical function, stiffness, functional performance, discomfort and psychological state, without difference among the groups, for 15 sessions. The authors infer that some positive US studies have low methodological quality and cite the use of other agents in the study may have masked the final effect and the study have a low sample size.

Bennell et al. (22) observed that both the multimodal therapy (taping, exercises, mobilization and massage) as the placebo US are effective in pain and patient global assessment, showing that only contact with the therapist can lead to positive changes in these patients.

Briefly, the 9 studies using ultrasound as a therapeutic strategy, 7 demonstrated positive effects on variables. There seems to be an indication that when the therapeutic ultrasound is isolated applied and compared to other therapies, show similar effectiveness to other therapeutic techniques. However, when comparing the different ultasound application forms (continuous or pulsed) is suggested that the pulsed application mode appears to be more effective for improvement in functionality variables.

\section{Neuromuscular electrical stimulation}

Scientific evidences used in this review show that the neuromuscular electrical stimulation (NMES) 
may be beneficial in patients with knee OA in variables such as pain $(6,23-26)$, physical function $(6$, $23,25)$, functional performance $(25,27)$, discomfort (6), daily living activities (6) and stiffness $(23,25)$. Furthermore, NMES seems to compensate physiological declines occurring in the muscle of patients, acting both in structure as in function of the quadriceps (23) by means of cross-sectional area increases (23, $27)$ and muscular strength $(23,25)$ and also in the improvement of voluntary muscle activation when combined with an exercise program (24). The parameters used in these studies $(6,23-27)$ varied from: $50-80 \mathrm{~Hz}, 100-400 \mu \mathrm{s}$, intensity: visible muscle contraction until the maximum tolerated, time on: 10 seconds, off time: 10-50 seconds 15-45 minutes per session, for 12-36 sessions.

Burch et al. (28) reported the efficacy of standard muscle stimulation $(50 \mathrm{~Hz}$ for $200 \mathrm{~ms}$ to $1500 \mathrm{~ms}$ each, intensity between $3.39 \mu$ s and $102.2 \mu$ s with an average production of $16.26 \mathrm{~mA}, 20$ minutes) in pain, stiffness, physical function and life quality when compared to low-intensity TENS $(0.2 \mathrm{~Hz}, 300 \mu \mathrm{s}, 0.5 \mathrm{~mA}$, 35 minutes), after 8 weeks. The standard muscle stimulation refers to stimulation characteristics: triphasic stimulation patterned based on the normal activation time of the quadriceps and hamstrings, during a high-level run. Before the standardized muscle stimulation, the group received IR current $(5.000 \mathrm{~Hz}$, pre-modulated between $1 \mathrm{~Hz}$ and $150 \mathrm{~Hz}$, tingling intensity, 15 minutes).

Disagreeing with the presented studies, PalmieriSmith et al. (29) didn't find gains in strength or activation in the quadriceps muscle after application of NMES $(2,500 \mathrm{~Hz} A C, 50$ bursts per second, maximum tolerated intensity, time on: 10 seconds, time off: 50 seconds, with 10 electrically induced contractions, for 12 sessions). The authors believe that the lack of effect may be due to low dysfunction of the quadríceps presented in the voluntaries of this study or due to the dosing of the intervention.

From 7 studies using neuromuscular electrical stimulation, 6 reported increases in physical and functional role. In studies that have shown positive effects of the application of NMES on the parameters analyzed, the NMES average parameters were: frequency $50 \mathrm{~Hz}$, pulse duration 200-250 microseconds and the maximum intensity tolerated by the patient. There seems to be an indication that the largest increases in muscle strength and functionality of the patients are inversely related to the deficit of muscle strength previous to treatment, in other words, the higher the deficit of muscle strength previous to treatment, the greater are the possibilities of improvement with use of NMES.

\section{Transcutaneous electrical nerve stimulation}

Law et al. (30) demonstrated the efficacy of different TENS parameters $(2 \mathrm{~Hz}, 100 \mathrm{~Hz}$ and $2 / 100 \mathrm{~Hz}, 576$ uS, $200 \mu$ s and $576 \mu \mathrm{s} / 200 \mu \mathrm{s}$, respectively, comfortable intensity levels, 40 minutes) in pain, functional performance and ROM. The authors found that TENS was superior to independent placebo application of current application parameters. Atamaz et al. (31) assessed the TENS efficacy $(80 \mathrm{~Hz}, 10-30 \mathrm{~mA})$, IF current, SWD, as well as the placebos interventions for each resource associated with exercise, in pain, physical function, functional performance and life quality, for 15 sessions. The authors found that the proposed therapies were effective in the variables analyzed in the study, and that the intake of analgesics was lower during the active interventions compared with placebos. In contrast, Cetin et al. (32) studied the effect of short-wave diathermy (SWD) $(27.12 \mathrm{MHz}$, field condenser technique, 15 minutes), transcutaneous electrical nerve stimulation (TENS) $(60-110 \mathrm{~Hz}$, $60 \mathrm{uS}$, maximum intensity without contraction 20 minutes), continuous US ( $1 \mathrm{MHz}, 1.5 \mathrm{~W} / \mathrm{cm}^{2}, 10$ minutes) associated with hot bag and exercise, compared with group of hot bag and exercise only or just exercise. The authors examined the effectiveness of SWD, TENS and continuous US interventions in pain, discomfort, physical function and MS. However, the best increments in the analyzed variables were obtained in SWD and TENS groups.

Studies $(33,34)$ have demonstrated the TENS effectiveness associated with physical exercise on muscle activation and a trend towards physical parameters improvement, when compared to isolated treatments. The parameters varied: $80-150 \mathrm{~Hz}, 140-$ $150 \mu \mathrm{s}$, sensory intensity, 60 minutes and 8 hours a day, 12-20 sessions.

Kolen et al. (35) demonstrated that for the TENS application $(80 \mathrm{~Hz}, 100 \mathrm{~ms}$, maximum tolerated intensity, 30-45 minutes) to be effective, it must be applied in locations with lower electrical skin resistance to reduce pain. However, when functionaly is the therapeutic goal, TENS can be applied on the greatest pain spots or random locations. 
Selfe et al. (36) studied the efficacy of adjuvant therapy of non-invasive interactive neurostimulation (NIN) in the "vitality" subscale of the SF-36 questionnaire and the overall patient evaluation, when compared to placebo, in 17 sessions for 20 to 30 minutes per session with progressive intensity. The NIN therapy refers to the application of TENS on acupuncture points, with low skin resistance. The NIN therapy also resulted in clinically significant reductions in pain, but without differences among groups, which may be explained by the small sample size and frequency of treatment have been less than ideal, since the frequency of weekly treatment decreased over study.

Only two studies $(37,38)$ analyzed the effectiveness of a single TENS session in knee OA. Vance et al.

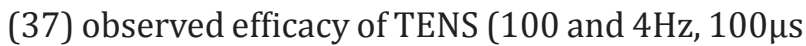
and intensity of $10 \%$ below the motor threshold, 40 50 minutes) and placebo treatment in pain at rest and during walking, however, only TENS groups increased the pressure pain threshold. The authors concluded that the placebo effect would possibly be reduced with more treatment sessions. Pietrosimone et al. (38) found that both the TENS $(150 \mathrm{~Hz}, 150 \mu \mathrm{s}$, sensory stimulation, 45 minutes) and the cryotherapy (2 bags of 50,721oz of ice for 20 minutes) has similar effects on quadriceps muscle activation immediately after the therapy application with no difference between the therapy groups.

Studies $(39,40)$ compared the effect of TENS and hyaluronic acid intra-articular injection in patients with knee OA. Chen et al. (39) observed greater efficiency of TENS $(3-20 \mathrm{~Hz}, 200 \mu \mathrm{s}$, maximum intensity tolerated, 20 minutes) in pain, discomfort and physical function compared to the injection of hyaluronic acid in 12 sessions. Meanwhile, Paker et al. (40) observed efficacy of TENS $(150 \mathrm{~Hz}, 20$ minutes to 1 hour, 15 sessions) and hyaluronic acid injection in pain, stiffness and physical function, with greater effect of hyaluronic acid in physical function. The different results between studies may be due to the types of electrodes and hyaluronic acid products that differed in the studies (39).

Disagreeing with the studies presented, Pietrosimone et al. (41) found no effect of TENS $(150 \mathrm{~Hz}, 150 \mu \mathrm{s}$, strong sensory intensity during each exercise session and a minimum of 8 hours a day) associated with exercise in angle and knee flexion peaks during pace. Moreover, the pain, stiffness, physical function, functional performance, muscle activation and MS have been improved in these patients after exercise regardless of the use of TENS. The absence of TENS effects can be attributed to insufficient sample size and the authors declare that the long treatment periods may be necessary to change these variables, since the quadriceps activation rate was higher in TENS group.

Briefly, TENS can be effective in reducing the pain perception and functionality in patients with knee osteoarthritis. For this, the electrodes application area can be a determining factor, because when the goal is to reduce pain, the electrodes must be applied in places of least resistance of the skin, but when the objective is to increase functionality, application of this feature in areas of greatest pain perception can be done. The use of TENS seems to be as or more effective than other analgesic therapies. From 11 articles included in this review, 10 articles evaluated the beneficial effects of TENS and 1 article didn't notice increases in comparison with the placebo group. However it is believed that the results can be explained due to the sample size and/or due to the insufficient treatment period.

Three studies examined the effectiveness of pulsed electrical stimulation (PES) in the perception of pain and self-reported health status in patients with knee OA $(42,43,44)$. Two studies $(42,43)$ verified efficiency of PES in pain, physical function, stiffness and selfreported health status of the patient, using the following parameters: $100 \mathrm{~Hz}, 2 \mathrm{~ms}$, subsensorial intensity for more than 6 hours/day. Furthermore, Fary et al. (42) found that these changes could be sustained for at least 16 weeks. Discordant with previous studies, Fary et al. (44) found no increased effectiveness of PES $(100 \mathrm{~Hz}, 4 \mathrm{~ms}, 7$ hours a day for 26 weeks) compared to placebo in pain, physical function, stiffness, lifge quality and exercise. The authors note that the sample may not be representative of the OA population, due to its characteristics.

According to the studies inserted in this review, it appears that three studies have tested the use of pulsed electrical stimulation in patients with knee osteoarthritis. Two studies have shown beneficial effects on pain, patient's global evaluation, physical function and joint stiffness. One study has shown no positive effects of pulsed electrical stimulation, but we believe the results can be explained because the sample may not be representative of the population of osteoarthritis. 
Low-power laser therapy

Kheshie et al. (45) demonstrated the effectiveness of high intensity laser (pulsed YAG laser 1250J in three phases: initial phase flow was adjusted to two applications of 710 and $810 \mathrm{~mJ} / \mathrm{cm}^{2}$, intermediate stage of $610 \mathrm{~mJ} / \mathrm{cm}^{2}$ and final phase, the same as first fluence for 45 minutes) and low intensity laser (BTL-5000 laser, As-Ga of 1250J, $50 \mathrm{~J} / \mathrm{cm}^{2}, 830 \mathrm{~nm}$, $1 \mathrm{Khz}, 800 \mathrm{~mW}$ for 32.33 minutes) on pain and physical functioning in 12 sessions. The authors demonstrated that both therapies are effective, but the high intensity laser appears to have best results.

Alfredo et al. (7) observed the low-intensity laser efficiency (As-Ga, $27 \mathrm{~J}$, being $3 \mathrm{~J}$ for 50 seconds per point, $904 \mathrm{~nm}, 700 \mathrm{~Hz}, 60 \mathrm{~mW}$ ) in pain, physical function and ROM compared to placebo. Agreeing with this study, Alghadir et al. (46) verified the efficacy of 8 low-intensity laser sessions (As-Ga, $50 \mathrm{~mW}, 850 \mathrm{~nm}$, 48J / $\mathrm{cm}^{2}$ with $6 \mathrm{~J} /$ point) in pain, physical function and functional performance compared to placebo condition. Hegedus et al. (47) assessed the efficacy of low level laser (Ga-Al-As, $50 \mathrm{~mW}, 830 \mathrm{~nm}, 6$ J per point, $48 \mathrm{~J} / \mathrm{cm}^{2}$ per session) in pain, ROM, pressure sensitivity and local microcirculation in 8 sessions compared the placebo condition

Al Rashoud et al. (48) demonstrated the effectiveness of low level laser (Ga-As-Al, $1.2 \mathrm{~J}$ for $40 \mathrm{sec}-$ onds per point, totaling $4 \mathrm{~J} / \mathrm{cm}^{2}, 830 \mathrm{~nm}, 30 \mathrm{~mW}$ ) applied on acupuncture points and associated with exercise for 9 sessions, in pain and physical function. Yurtkuran et al. (49) also evaluated the laser effectiveness (As-Ga $4 \mathrm{~mW}, 904 \mathrm{~nm}$ and $0.48 \mathrm{~J}$ per session, $10 \mathrm{~mW} / \mathrm{cm} 2$ ) on acupuncture points and observed improvement only in the edema seen by knee circunference compared to the placebo group, in 10 sessions. The authors point out that the dose adopted in the study was lower than that recommended by the World Association of Laser Therapy, which may have influenced the results.

Disagreeing with the presented studies, Tascioglu et al. (50) found no efficacy in pain, physical function and stiffness in the different therapies with low intensity laser: Group 1: Laser for 2 minutes per point (Ga-Al-As, 50mW, 830nm, 3J per point, totaling 15J per session, a total of 10 minutes); Group 2: Laser for 1 minute per point (Ga-Al-As, $50 \mathrm{~mW}, 830 \mathrm{~nm}, 1.5 \mathrm{~J}$ per session, totaling 5 minutes); and Group 3: placebo laser. The authors afirm that the results can be justified by the laser mode, dosages and wavelength adopted in the study that generated an ineffective treatment.

Of the 6 studies inserted in this review, 5 proved to be effective to improve pain and function in patients with knee osteoarthritis. From the analysis of the results presented, it is believed that the therapy with the low intensity laser can improve pain, range of motion and functional performance, especially compared to the placebo condition.

\section{Study limitations}

The studies used in this review demonstrate results variations according to each physical modality and should be carefully considered by professionals to choose the best physical modality for each individual. Furthermore, there are some limitations in this study, among which can be highlighted: 1) The inclusion of studies that evaluated the physical modalities associated with other forms of intervention, preventing conclude what the real effect of the isolated application mode is. Though, it is emphasized that such modalities are complementary and are not clinically used in isolation; 2) The difficulty of establishing a treatment protocol facing the results and divergent application methods, the lack of information in some studies, besides the scarcity of published articles.

\section{Conclusion}

The studies showed in this review demonstrated variations on the benefits of physical modalities with respect to the used parameters, frequency of treatment, and application sites. Although, it was found based on the last 10 years literature, using high-designed studies, these methods are effective for this population, improving their symptoms and signs. For more treatment effectiveness, these interventions should be adjusted depending on the goal that you want to achieve with each patient, with specific protocols for each clinical condition.

\section{References}

1. Goldring MB, Goldring SR. Osteoarthritis. J Cell Physiol. 2007;213(3):626-34. 
2. Zacaron KAM, Days JMD, Abreu NS, Dias RC. Physical Activity Levels, Pain and Swelling and Their Relationships with Knee Muscle Dysfunction in Elderly People with Osteoarthritis. Rev Bras Fisioter. 2006;10(3):279-84.

3. Michael JW-P, Schlüter-Brust KU, Eysel P. The epidemiology, Etiology, Diagnosis, and Treatment of osteoarthritis of the knee. Dtsch Arztebl Int. 2010;107(9):152-62.

4. Jamtvedt G, Dahm KT, Christie A, Moe HR, Haavardsholm E, Holm I, et al. Physical therapy interventions for Patients with osteoarthritis of the knee: an overview of systematic reviews. Phys Ther. 2008;88(1):123-36.

5. Mascarin NC, Vancini RL, ML Andrade, Magellan EP, CA De Lira, Coimbra IB. Effects of kinesiotherapy, electrotherapy and ultrasound in management of bilateral knee osteoarthritis: prospective clinical trial. BMC Musculoskelet Disord. 2012;13:182.

6. Imoto AM, Peccin MS, Teixeira LE, Silva KNGS, Abrahão M, Trevisani VFM. Neuromuscular electrical stimulation is effective for Improving pain, function and activities of daily living of knee osteoarthritis patients? A randomized clinical trial. Sao Paulo Med J. 2013;131(2):80-7.

7. Alfredo PP, Bjordal JM, SH Dreyer, Meneses SRF, Zaguetti G, Ovanessian V, et al. Efficacy of low level laser therapy associated with exercises in knee osteoarthritis: a randomized double-blind study. Clin Rehabil. 2012;26(6):523-33.

8. Kawasaki T, Inoue K, Ushiyama T, Fukuda S. [Assessment of the American College of Rheumatology criteria for the classification and reporting of osteoarthritis of the knee]. Ryumachi. 1998;38(1):2-5. Japanese.

9. Maher CG, Sherrington C, Herbert RD, Moseley AM, Elkins M. Reliability of the PEDro Scale for rating quality of randomized controlled trials. Phys Ther. 2003;83(8):713-21.

10. Ackerman I. Western Ontario and McMaster Universities Osteoarthritis Index (WOMAC). Aust J Physiother. 2009;55(3):213.

11. Verhagen AP, de Vet HC, de Bie RA, Kessels AG, Boers M, Bouter LM, et al. The Delphy list: the criteria list for quality assessment of randomized clinical trials for conducting systematic reviews developed by Delphi Consensus. J Clin Epidemiol. 1998;51(12):1235-41.
12. Moseley AM, Herbert RD, Sherrington C, Maher CG. Evidence for physiotherapy practice: a survey of the Physiotherapy Evidence Database (PEDro). Aust J Physiother. 2002;48(1):43-9.

13. Tascioglu F, Kuzgun S, Armagan O, Ogutler G. Shortterm effectiveness of ultrasound therapy in knee osteoarthritis. J Int Med Res. 2010;38(4):1233-42.

14. MH Huang, Lin YS, Lee CL, Yang RC. Use of ultrasound to increase effectiveness of isokinetic exercise for knee osteoarthritis. Arch Phys Med Rehabil. 2005;86(8):1545-51.

15. Huang MH, Yang RC, Lee CL, Chen TW, Wang MC. Preliminary results of integrated therapy for patients with knee osteoarthritis. Arthritis Rheum. 2005;53(6):812-20.

16. Cakir S, Hepguler S, Ozturk C, Korkmaz M, Isleten B, Atamaz FC. Efficacy of therapeutic ultrasound for the management of knee osteoarthritis: a randomized, controlled, and double-blind study. Am J Phys Med Rehabil. 2014;93(5):405-12.

17. Ozgönenel L, Aytekin E, Durmuşoglu G. A doubleblind trial of clinical effects of therapeutic ultrasound in knee osteoarthritis. Ultrasound Med Biol. 2009;35(1):44-9.

18. Luksurapan W, Boonhong J. Effects of phonophoresis of piroxicam and ultrasound on symptomatic knee osteoarthritis. Arch Phys Med Rehabil. 2013;94(2):250-5.

19. Külcü DG, Gülşen G, Altunok E. Short-term efficacy of pulsed electromagnetic field therapy on pain and functional level in knee osteoarthritis: a randomized controlled study. Turk J Rheumatol. 2009;24(3):144-8.

20. Ozgüçlü E, Cetin A, Cetin M, Calp E. Additional effect of pulsed electromagnetic field therapy on knee osteoarthritis treatment: a randomized, placebo-controlled study. Clin Rheumatol. 2010;29(8):927-31.

21. Ulus Y, Tander B, Akyol Y, Durmus D, Buyukakincak $\mathrm{O}$, Gul U, et al. Therapeutic ultrasound versus sham ultrasound for the management of patients with knee osteoarthritis: a double-blind randomized controlled clinical study. Int J Rheum Dis. 2012;15(2):197-206. 
22. Bennell KL, Hinman RS, Metcalf BR, Buchbinder R, McConnell J, McColl G, et al. Efficacy of physiotherapy management of knee joint osteoarthritis: a randomized, double blind, placebo controlled trial. Ann Rheum Dis. 2005;64(6):906-12.

23. Vaz MA, Baroni BM, Geremia JM, Lanferdini FJ, Mayer A, Arampatziz A, et al. Neuromuscular electrical stimulation (NMES) reduces structural and functional losses of quadriceps muscle and improves health status in patients with knee osteoarthritis. J Orthop Res. 2013;31(4):511-6.

24. Elboim-Gabyzon M, Rozen N, Laufer Y. Does neuromuscular electrical stimulation enhance the effectiveness of an exercise program in subjects with knee osteoarthritis? A randomized controlled trial. Clin Rehabil. 2013;27(3):246-57.

25. Durmuş D, Alayli G, Cantürk F. Effects of quadriceps electrical stimulation program on clinical parameters in the patients with knee osteoarthritis. Clin Rheumatol. $2007 ; 26(5): 674-8$.

26. Gaines JM, Metter EJ, Talbot LA. The effect of neuromuscular electrical stimulation on arthritis knee pain in older adults with osteoarthritis of the knee. Appl Nurs Res. 2004;17(3):201-6.

27. Bruce-Brand RA, Walls RJ, Ong JC, Emerson BS, O’Byrne JM, Moyna NM. Effects of home-based resistance training and neuromuscular electrical stimulation in knee osteoarthritis: a randomized controlled trial. BMC Musculoskelet Disord. 2012;13:118.

28. Burch FX, Tarro JN, Greenberg JJ, Carroll WJ. Evaluating the benefits of patterned stimulation in the treatment of osteoarthritis of the knee: a multi-center, randomized, single-blind, controlled study with an independent evaluator masked. Osteoarthritis Cartilage. 2008;16(8):865-72.

29. Palmieri-Smith RM, Thomas AC, Karvonen-Gutierrez $C$, Sowers M. The clinical trial of neuromuscular electrical stimulation in Improving quadriceps muscle strength and activation Among women with mild and moderate osteoarthritis. Phys Ther. 2010;90(10):1441-52.

30. Law PP, Cheing GL. Optimal stimulation frequency of transcutaneous electrical nerve stimulation on people with knee osteoarthritis. J Rehabil Med. 2004;36(5):220-5.
31. Atamaz FC, Durmaz B, Baydar M, Demircioglu OY, Iyiyapici A, Kuran B, et al. Comparison of the efficacy of transcutaneous electrical nerve stimulation, interferential currents, and shortwave diathermy in knee osteoarthritis: a double-blind, randomized, controlled, multicenter study. Arch Phys Med Rehabil. 2012;93(5):748-56.

32. Cetin N, Aytar A, Atalay A, Akman MN. Comparing hot pack, short-wave diathermy, ultrasound, and TENS on isokinetic strength, pain and functional status of women with osteoarthritic knees: a single-blind, randomized, controlled trial. Am J Phys Med Rehabil. 2008;87(6):443-51.

33. Cheing GL, Hui-Chan CW. Would the addition of TENS to exercise training produce better physical performance outcomes in people with knee osteoarthritis than either intervention alone? Clin Rehabil. 2004;18(5):487-97.

34. Pietrosimone BG, Saliba SA, Hart JM, Hertel J, Kerrigan DC, Ingersoll CD. Effects of transcutaneous electrical nerve stimulation and therapeutic exercise on quadriceps activation in people with tibiofemoral osteoarthritis. J Orthop Sports Phys Ther. 2011;41(1):4-12.

35. Kolen AF, de Nijs RN, Wagemakers FM, Meier AJ, Johnson MI. Effects of spatially targeted transcutaneous electrical nerve stimulation using an electrode array that measures skin resistance on pain and mobility in patients with osteoarthritis in the knee: a randomized controlled trial. Pain. 2012;153(2):373-81.

36. Selfe TK, Bourguignon C, Taylor AG. Effects of noninvasive neurostimulation on interactive Symptoms of osteoarthritis of the knee: a randomized, shamcontrolled pilot study. J Altern Complement Med. 2008;14(9):1075-81.

37. Vance CG, Rakel BA Blodgett NP, DeSantana JM, Amendola A, Zimmerman MB, et al. Effects of transcutaneous electrical nerve stimulation on pain, pain sensitivity, and function in people with knee osteoarthritis: a randomized controlled trial. Phys Ther. 2012;92(7):898-910.

38. Pietrosimone BG, Hart JM, Saliba SA, Hertel J, Ingersoll CD. Immediate effects of transcutaneous electrical nerve stimulation and focal knee joint cooling on quadriceps activation. Med Sci Sports Exerc. 2009;41(6):1175-81. 
39. Chen WL, Hsu WC, Lin YJ, Hsieh LF. Comparison of intra-articular hyaluronic acid injections with transcutaneous electric nerve stimulation for the management of knee osteoarthritis: a randomized controlled trial. Arch Phys Med Rehabil. 2013;94(8):1482-9.

40. Paker N, Tekdös D, Kesiktas N, Soy D. Comparison of the therapeutic efficacy of TENS versus intra-articular hyaluronic acid injection in Patients with knee osteoarthritis: a prospective randomized study. Adv Ther. 2006;23(2):342-53.

41. Pietrosimone BG, Saliba SA, Hart JM, Hertel J, Kerrigan DC, Ingersoll CD. Effects of disinhibitory transcutaneous electrical nerve stimulation and therapeutic exercise on sagittal plane peak knee kinematics and kinetics in people with knee osteoarthritis during gait: a randomized controlled trial. Clin Rehabil. 2010;24(12):1091-101.

42. Fary RE, Briffa NK, Briffa TG. Effectiveness of pulsed electrical stimulation in the management of osteoarthritis of the knee: three case reports. Physiother Theory Pract. 2009;25(1):21-9.

43. Garland D, Holt P Harrington JT, Caldwell J, Zizic T, Cholewczynski J. A 3-month, randomized, doubleblind, placebo-controlled study to evaluate the safety and efficacy of a highly optimized, capacitively coupled, pulsed electrical stimulator in Patients with osteoarthritis of the knee. Osteoarthritis Cartilage. 2007;15(6):630-7.

44. Fary RE, Carroll GJ, Briffa TG, Briffa NK. The effectiveness of pulsed electrical stimulation in the management of osteoarthritis of the knee: results of a double-blind, randomized, placebo-controlled, repeated-measures trial. Arthritis Rheum. 2011;63(5):1333-42.
45. Kheshie AR, Alayat MS, Ali MM. High-intensity versus low-level laser therapy in the treatment of Patients with knee osteoarthritis: a randomized controlled trial. Lasers Med Sci. 2014;29(4):1371-6.

46. Alghadir A, Omar MT, Al-Askar AB, Al-Muteri NK. Effect of low-level laser therapy in Patients with chronic knee osteoarthritis: a single-blinded randomized clinical study. Lasers Med Sci. 2014;29(2):749-55.

47. Hegedus B, Viharos L, Gervain M, Gálfi M. The effect of low-level laser in knee osteoarthritis: a double-blind, randomized, placebo-controlled trial. Photomed Laser Surg. 2009;27(4):577-84

48. Al Rashoud AS, Abboud RJ, Wang W, Wigderowitz C. Efficacy of low-level laser therapy applied at acupuncture points in knee osteoarthritis: a doubleblind randomized comparative trial. Physiotherapy. 2014;100(3):242-8.

49. Yurtkuran M, Alp A, Konur S, Özçakir S, Bingol U. Laser acupuncture in knee osteoarthritis: a double-blind, randomized controlled study. Photomed Laser Surg. 2007;25(1):14-20.

50. Tascioglu F, Armagan O, Y Tabak, Corapci I Oner C. Low power laser treatment in patients with knee osteoarthritis. Swiss Med Wkly. 2004;134(17-18):254-8.

Received in 08/31/2015 Recebido em 31/08/2015

Approved in 07/28/2016 Aprovado em 28/07/2016 